\title{
Generando gráficos del COVID-19 EN LA RePÚblica Dominicana USANDo R: EL PaQUETE DRCOVIDPLOTS
}

\author{
Fidel Morla ${ }^{a}$ y Johan Rosa ${ }^{b}$ \\ Recibido: 1/5/2020 A Aprobado: 31/7/2020
}

Cómo citar: Morla, F., \& Rosa, J. (2020). Generando gráficos del COVID-19 en la República Dominicana usando R: el paquete drcovidplots. Ciencia, Economía y Negocios, 4(2), 33-50. Doi: https://doi.org/10.22206/ ceyn.2020.v4i2.pp33-50

\section{Resumen}

El paquete drcovidplots en $R$ tiene diversas funciones para mostrar datos de forma gráfica del COVID-19 en República Dominicana. La información nacional proviene del Ministerio de Salud Pública y Asistencia Social, mientras que la información a nivel global es extraida de Worldometer ${ }^{\text {. }}$.

Palabras clave: COVID19; programación; R-Studio; análisis gráfico.

Códigos JEL: C88, Y10, I10.

a Banco Central de la República Dominicana, Santiago de los Caballeros, República Dominicana. Correo-e: f.morla@bancentral.gov.do

b Banco Central de la República Dominicana, Santiago de los Caballeros, República Dominicana. Correo-e: j.rosa@bancentral.gov.do

1. https://www.worldometers.info/

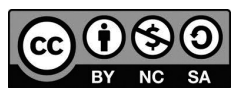

Esta obra está bajo licencia internacional Creative Commons Atribución-NoComercial-CompartirIgual 4.0 Internacional 


\title{
GeNERATING CHARTS TO ANALYZE COVID-19 IN THE DOMINICAN REPUBLIC USING R: THE DRCOVIDPLOTS PACKAGE
}

\author{
Fidel Morla ANd Johan Rosa
}

Received: 1/5/2020 • Approved: 31/7/2020

\begin{abstract}
The R package drcovidplots has various functions to display graphically $C O-$ VID-19 data in the Dominican Republic. Domestic information is mined from the Ministry of Public Health and Social Assistance, while global information is extracted from Worldometer.
\end{abstract}

Keywords: COVID19; programming; R-Studio; graphic analysis.

JEL Codes: C88, Y10, I10. 


\title{
1. Introducción
}

En estadística, una representación gráfica es una visualización de datos en forma de diagrama o gráfico. Es decir, representa un conjunto de datos de manera significativa. Además, observar información de forma gráfica puede ayudar a tomar mejores decisiones (Nussbaumer, 2015).

Usualmente, los economistas utilizan el método de representación gráfica ya que es efectivo para mostrar y resumir grandes cantidades de datos, y es útil para mostrar tendencias, patrones y relaciones entre variables. Adicionalmente, se pueden tomar decisiones comparando el valor o el costo, discutiendo algún informe de cómo se presentó una situación específica en el pasado respecto al presente. Considerando que este método facilita la interpretación de los datos, tiene muchas ventajas en los campos de la estadística y la economía. Por esto, los gráficos y cuadros son actualmente componentes fundamentales de la investigación moderna y la presentación de informes.

De esta forma, teniendo en cuenta la situación pandémica que vive el mundo producto del coronavirus y su consecuente enfermedad COVID-19, este documento presenta una herramienta de análisis gráfico con información del Ministerio de Salud Pública y Asistencia Social de la República Dominicana (MSPAS). Este instrumento es el paquete de R llamado drcovidplots, el cual simplifica esta tarea con las funciones que incluye. Solo se necesita un conocimiento básico de programación en $\mathrm{R}$ para ejecutar el paquete y reproducir los gráficos.

\section{Instalación}

\section{La versión de desarrollo de drcovidplots puede instalarse desde GitHub desde el indicador de la consola $R$ con:}

\author{
install.packages("devtools") \\ devtools::install_github(“fidelmorla/drcovidplots") \\ library("drcovidplots") \\ Todas las dependencias faltantes deben instalarse automática- \\ mente.
}




\section{Uso}

\subsection{Cargando la información}

Para ejemplificar el uso de drcovidplots, utilizamos la información disponible en el boletín especial número 91 del MSPAS. Además, debe mencionarse que la información está en archivos con valores separados por comas en la página del repositorio en Github ${ }^{1}$. Por esta razón, es imprescindible tener conexión a internet a la hora de cargar la información con la función que se muestra más abajo.

Para cargar estos datos, se debe correr el siguiente código en la consola de R:

load_data_covid_dr()

Esta función creará los siguientes objetos:

Tabla 1. Objetos creados por la función load_data_covid_dr()

\begin{tabular}{|c|c|c|}
\hline Objeto & $\begin{array}{c}\text { Clase del } \\
\text { objeto }\end{array}$ & Contenido \\
\hline data_cum & \multirow{5}{*}{ data.frame } & Información agregada por fecha \\
\hline data_density & & Densidad poblacional por provincia \\
\hline data_province & & Positivos, muertes, recuperados y pruebas por provincia \\
\hline data_sex & & Positivos por sexo al último reporte disponible \\
\hline data_type & & Distribución por tipo de aislamiento o estado de los positivos \\
\hline East & \multirow{5}{*}{ character } & Vector con las abreviaciones para las provincias de la Región Este \\
\hline Metropolitan & & $\begin{array}{l}\text { Vector con las abreviaciones para las provincias de la Región } \\
\text { Metropolitana }\end{array}$ \\
\hline North & & $\begin{array}{c}\text { Vector con las abreviaciones para las provincias de la Región } \\
\text { Norte }\end{array}$ \\
\hline South & & Vector con las abreviaciones para las provincias de la Región Sur \\
\hline $\mathrm{NE}$ & & Vector con la abreviación para la fila de No especificado \\
\hline rep_actual & integer & $\begin{array}{l}\text { Número entero indicando el número del último boletín espe- } \\
\text { cial del MSPAS }\end{array}$ \\
\hline
\end{tabular}

Fuente: elaboración propia.

1 https://github.com/fidelmorla/drcovidplots/tree/master/csv data 


\subsection{Funciones}

La utilización de las funciones es relativamente sencilla. Todas las funciones tienen los siguientes dos argumentos:

Tabla 2. Argumentos más recurrentes en las funciones

\begin{tabular}{|c|c|}
\hline Argumento & Descripción \\
\hline \multirow{2}{*}{ saveplot } & Lógico. ¿Debería guardar el objeto ggplot en .GlobalEnv? \\
& Predeterminado FALSE. \\
\hline \multirow{2}{*}{ savepng } & Lógico. ¿Debería guardar una versión png del gráfico? \\
& Predeterminado FALSE. \\
\hline
\end{tabular}

Fuente: elaboración propia.

Las únicas excepciones son: la función g_cases_province(), la cual incluye un argumento adicional n_province para determinar el número de provincias con las cuales se generará el gráfico. Esta función representa gráficamente, en orden descendente, las provincias dadas en n_province. De forma predeterminada, la función grafica las quince provincias con más casos de COVID-19 partiendo de la información del último boletín del MSPAS.

La otra función que cuenta con otros argumentos es la g_map_ covid(). Esta cuenta con:

i) el argumento date, para indicar la fecha para introducir en el mapa, predeterminado la última fecha disponible ("latest"),

ii) el argumento variable, para indicar cuál variable utilizar. Tiene tres posibilidades: "Cases", "Deaths" y "Recovered",

iii) el argumento by_inhabitants, una variable lógica que indica si crear el mapa teniendo en cuenta la población de cada provincia. De forma predeterminada es "TRUE". 


\subsection{Ejemplos}

Ahora ejemplificaremos el uso de algunas funciones. Debe mencionarse que para el siguiente ejercicio se asume que se ejecutaron las funciones descritas en la parte 3.1.

Por ejemplo, utilizaremos la función g_cases_province() con dos opciones para el número de provincias, dígase 11 y 22.

$>$ g_cases_province $\left(\mathrm{n} \_\right.$province $=11$, savepng $=$ TRUE $)$

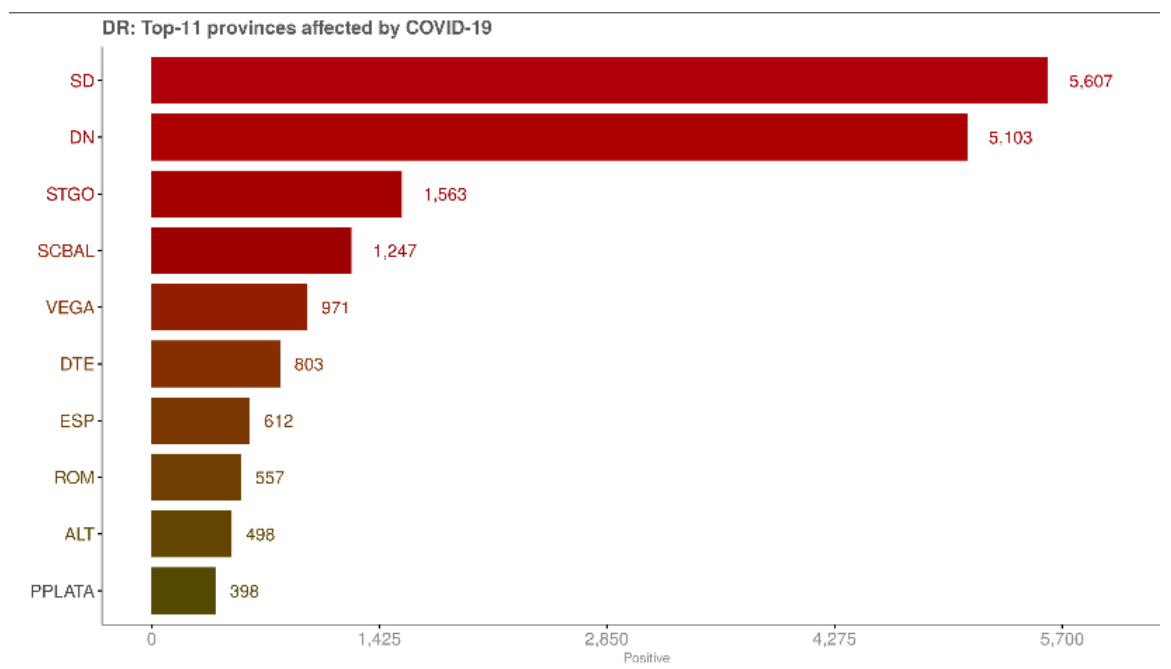

Figura 1. Las 11 provincias con mayor cantidad de casos de COVID19 en RD

Fuente: Información disponible en el boletín especial del MSPAS número 91.

Nota. Se utiliza el argumento de guardado en png en todas las funciones para generar el gráfico 


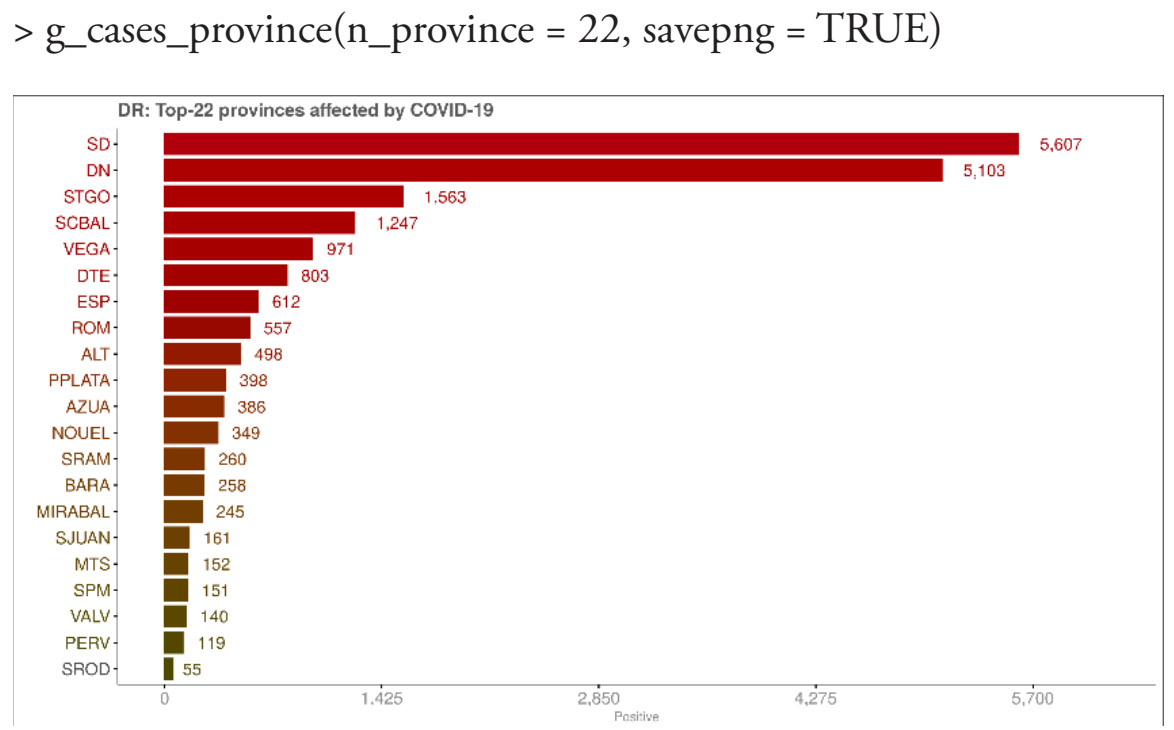

Figura 2. Las 22 provincias con mayor cantidad de casos de COVID19 en RD. Fuente: Información disponible en el boletín especial del MSPAS número 91. Nota. Se utiliza el argumento de guardado en png en todas las funciones para generar el gráfico

Otras funciones:

$>$ g_sex $($ savepng $=$ TRUE $)$

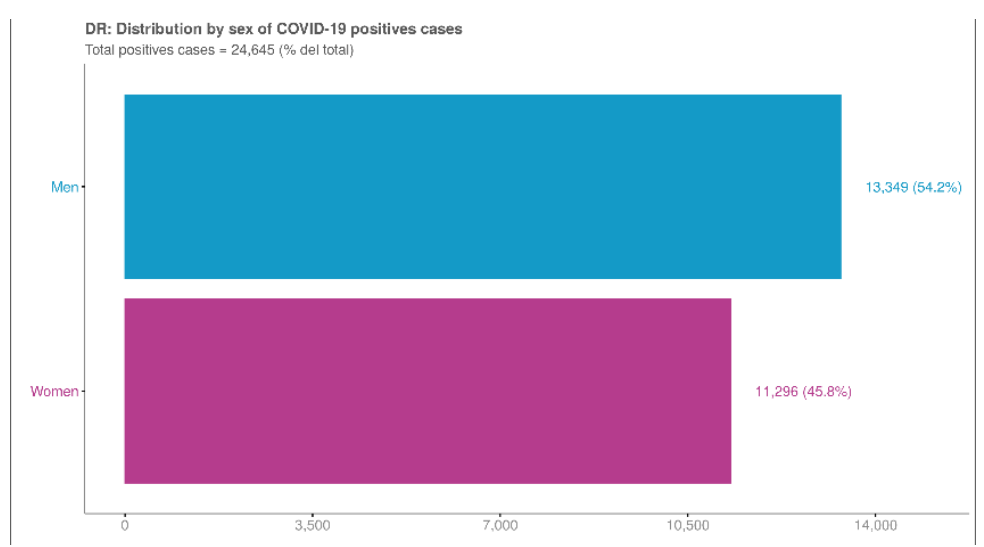

Figura 3. Distribución por sexo de los casos positivos de COVID19

Fuente: Información disponible en el boletín especial del MSPAS número 91.

Nota. Se utiliza el argumento de guardado en png en todas las funciones para generar el gráfico 
$>$ g_recovered_province $($ savepng $=$ TRUE $)$

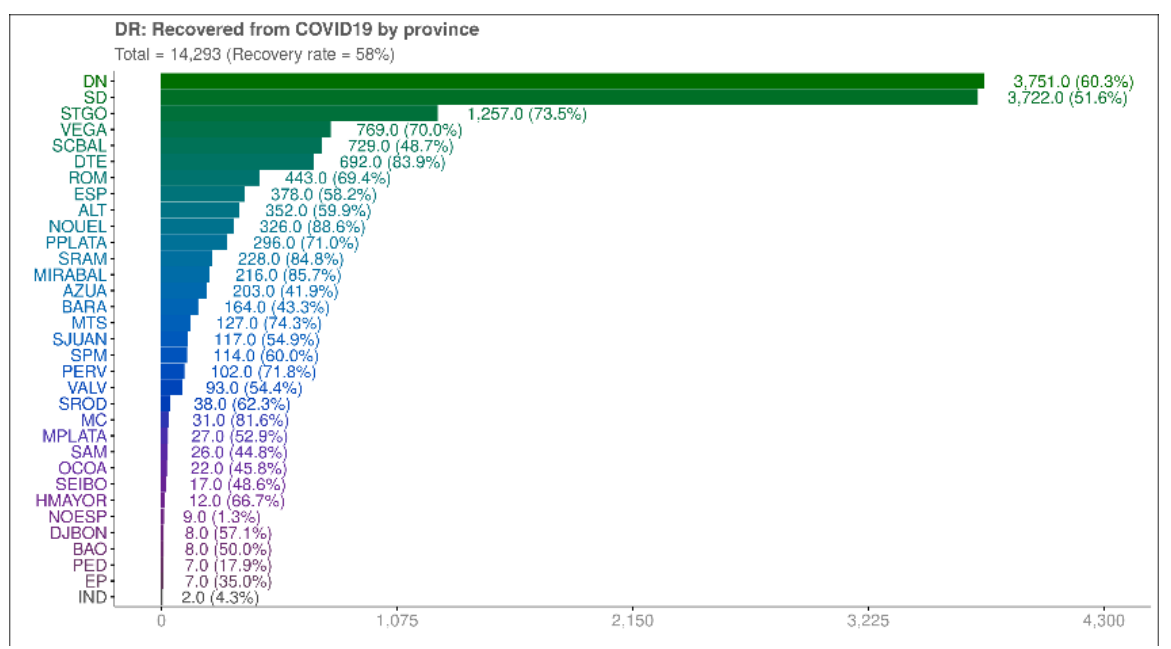

Figura 4. Recuperados de COVID19 por provincia

Fuente: Información disponible en el boletín especial del MSPAS número 91.

Nota. Se utiliza el argumento de guardado en png en todas las funciones para generar el gráfico

$>$ g_death(savepng $=$ TRUE $)$

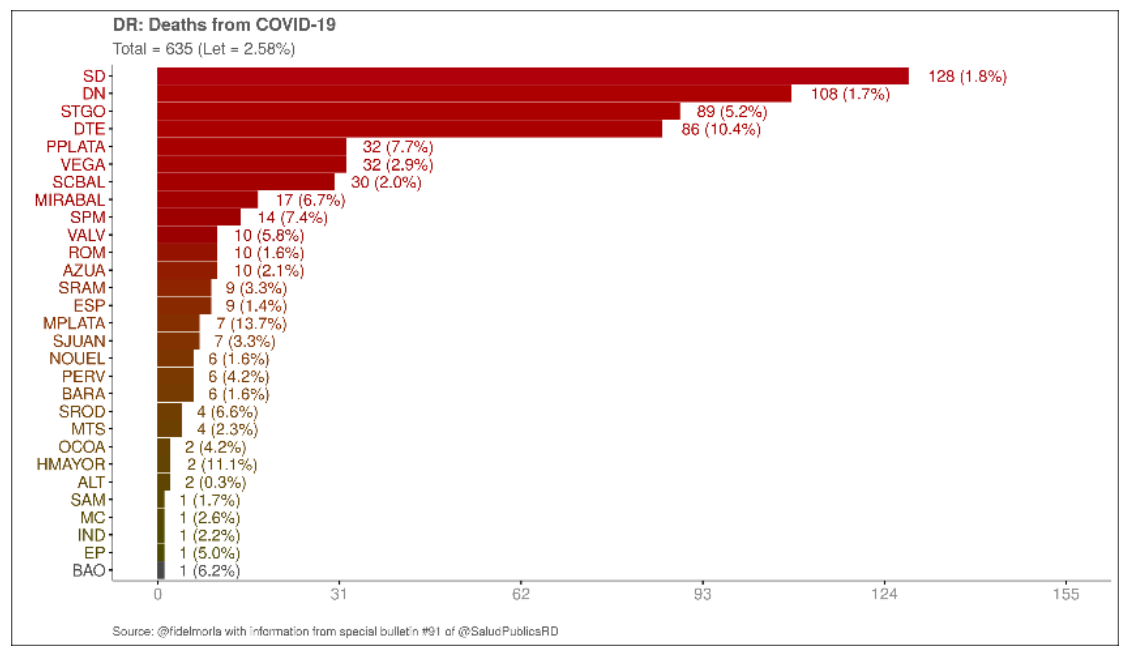

Figura 5. Decesos por COVID19

Fuente: Información disponible en el boletín especial del MSPAS número 91.

Nota. Se utiliza el argumento de guardado en png en todas las funciones para generar el gráfico 


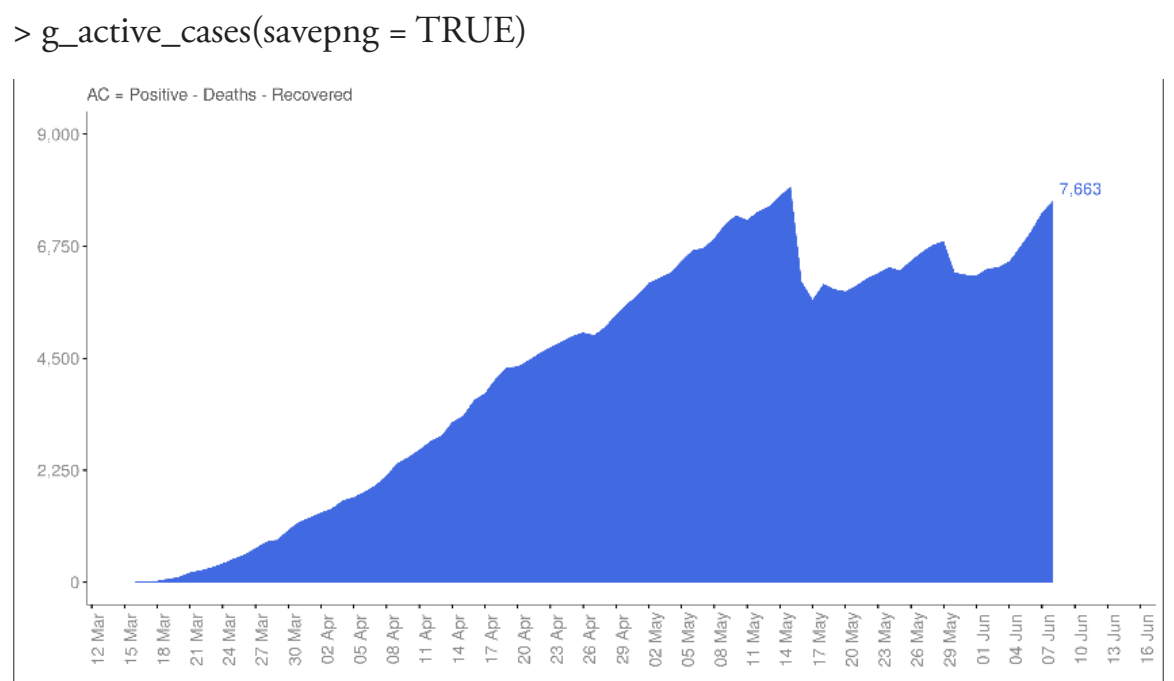

Figura 6. Casos activos de COVID19

Fuente: Información disponible en el boletín especial del MSPAS número 91.

Nota. Se utiliza el argumento de guardado en png en todas las funciones para generar el gráfico

$>$ g_positive_province $($ savepng $=$ TRUE $)$

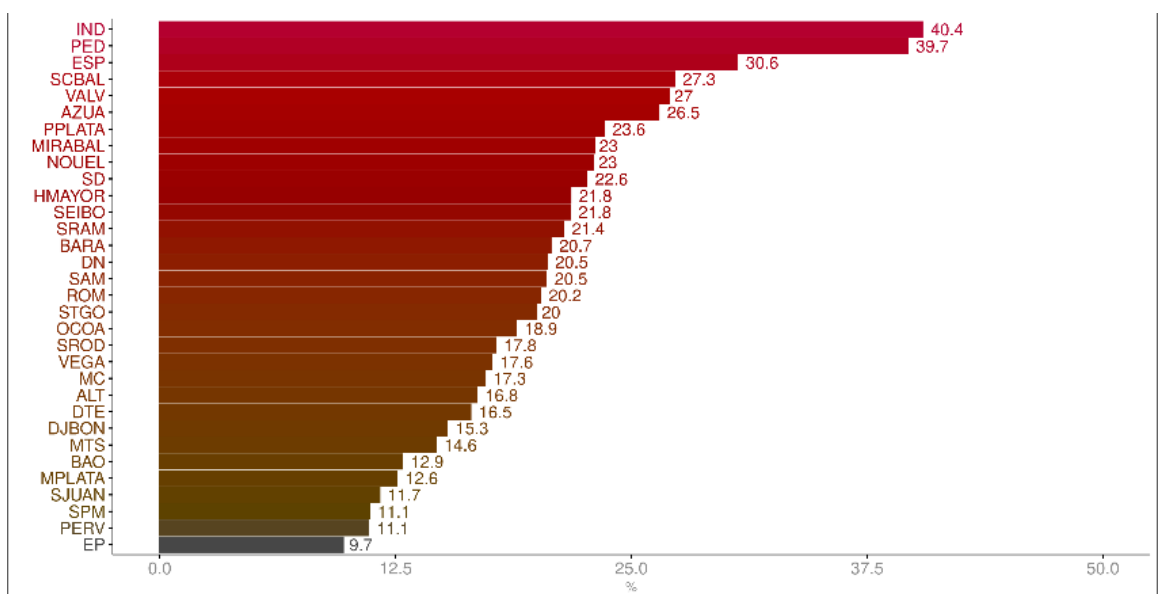

Figura 7. Positividad de COVID19 por provincia

Fuente: Información disponible en el boletín especial del MSPAS número 91.

Nota. Se utiliza el argumento de guardado en png en todas las funciones para generar el gráfico. La positividad es el ratio entre los casos positivos y el número de pruebas realizadas. 
$>$ g_tests (savepng $=$ TRUE)

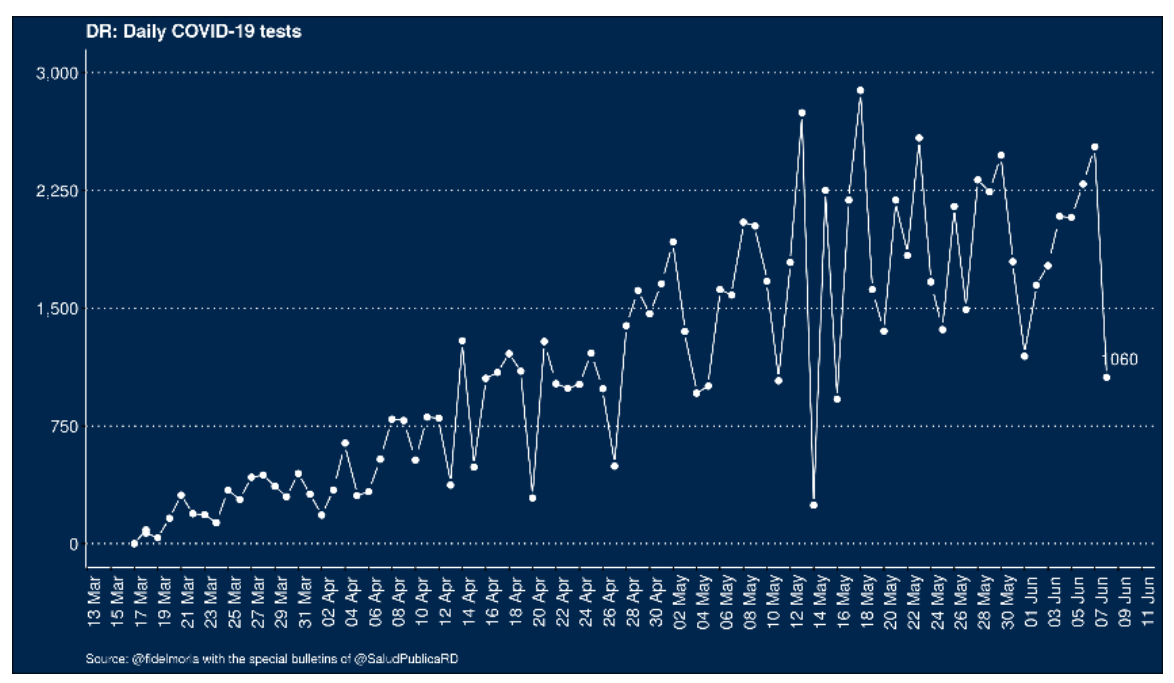

Figura 8. Pruebas diarias de COVID19

Fuente: Información disponible en el boletín especial del MSPAS número 91.

Nota. Se utiliza el argumento de guardado en png en todas las funciones para generar el gráfico.

Esta última función genera un total de cuatro gráficos, todos concernientes a las pruebas realizadas:

i. Pruebas diarias

ii. Pruebas acumuladas

iii. Pruebas diarias por cada millón de habitantes

iv. Pruebas acumuladas por cada millón de habitantes 
> g_map_covid $($ variable $=$ 'Cases', savepng $=$ TRUE $)$

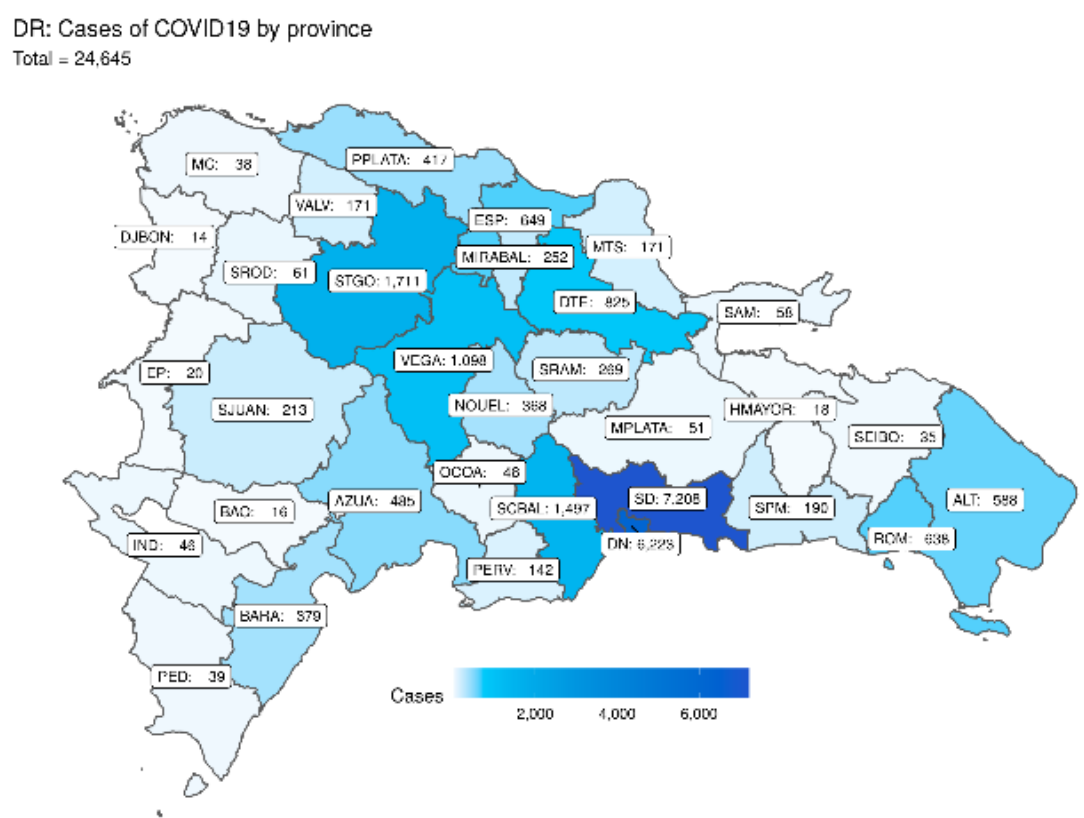

Figura 9. Mapa de casos activos de COVID19 por provincia

Fuente: Información disponible en el boletín especial del MSPAS número 91.

Nota. Se utiliza el argumento de guardado en png en todas las funciones para generar el gráfico. 
$>$ g_map_covid $($ variable $=$ 'Deaths', savepng $=$ TRUE $)$

DR: Deaths by COVID 19 by province

Total $=635$

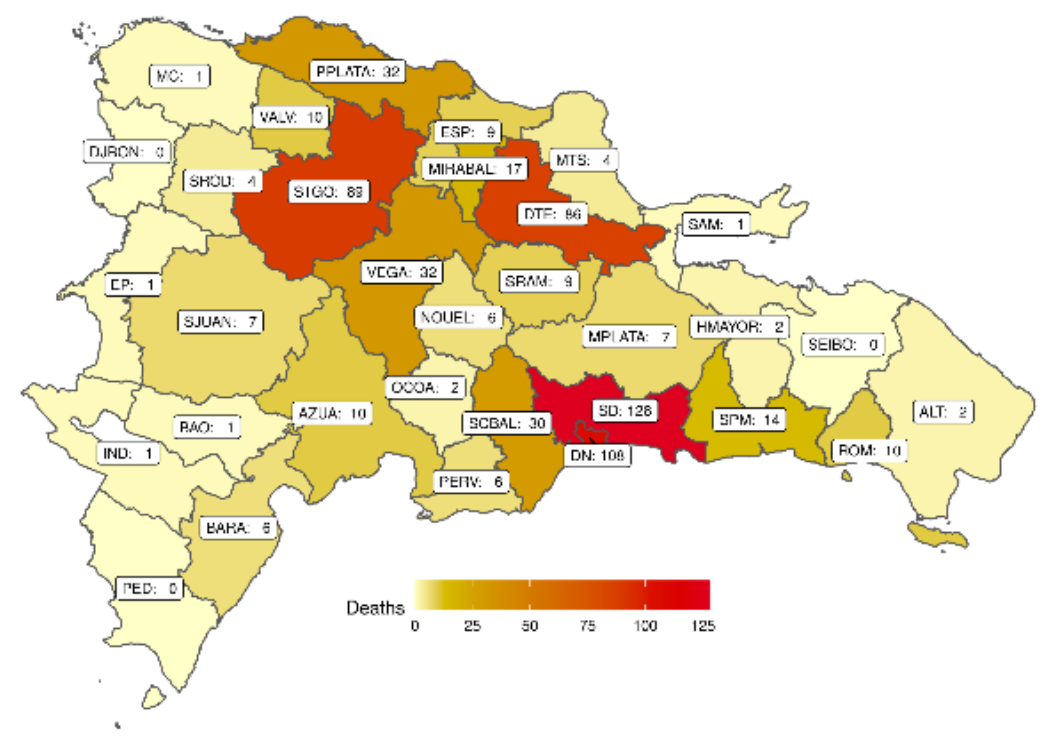

Figura 10. Mapa de decesos por COVID19 por provincia

Fuente: Información disponible en el boletín especial del MSPAS número 91.

Nota. Se utiliza el argumento de guardado en png en todas las funciones para generar el gráfico 
> g_map_covid $($ variable $=$ 'Recovered', savepng $=$ TRUE $)$

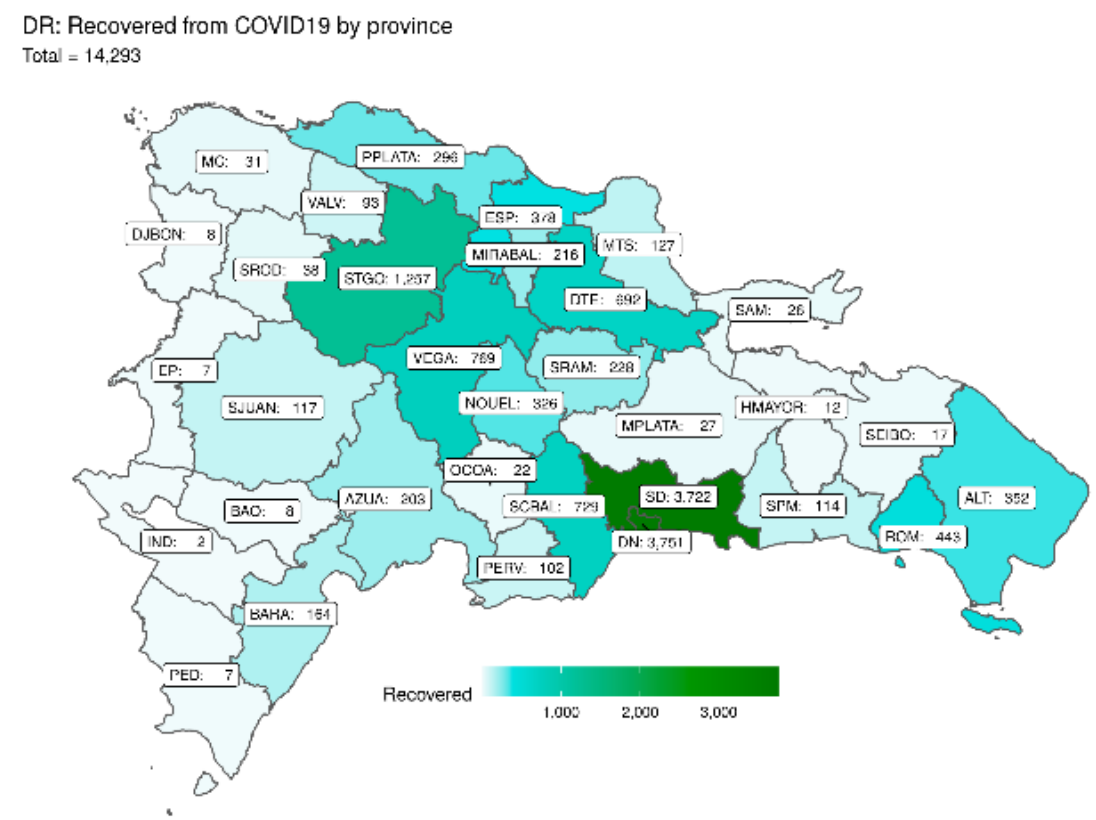

Figura 10. Mapa de recuperados de COVID19 por provincia

Fuente: Información disponible en el boletín especial del MSPAS número 91.

Nota. Se utiliza el argumento de guardado en png en todas las funciones para generar el gráfico

\section{Conclusión}

Este documento se dedica principalmente a explicar el funcionamiento del paquete drcovidplots, el cual proporciona varias funciones para graficar diferentes variables sobre la situación del COVID19 en República Dominicana. Esta herramienta de análisis gráfico tiene 27 funciones que utilizan información de los boletines especiales del MSPAS. Cabe la posibilidad de que la herramienta continúe evolucionando, a medida que surja más información del COVID19 en República Dominicana. Por tal razón, siempre recomendamos actualizar el paquete ocasionalmente. 
Los códigos del paquete están disponibles en el enlace https:// github.com/fidelmorla/drcovidplots. Además, los usuarios que utilicen el paquete y encuentren problemas o dificultades pueden reportarlas en la página https:/github.com/fidelmorla/drcovidplots/issues.

\section{Cita oficial}

Para citar el paquete "drcovidplots" en publicaciones, use:

Morla, F. (2020). drcovidplots: Plots for COVID19 data from Dominican Republic. R package version 0.1.11. https://github.com/ fidelmorla/drcovidplots.

\section{Referencias}

Arnold, J. B. (2019). ggthemes: Extra Themes, Scales and Geoms for 'ggplot2'. R package version 4.2.0. Recuperado de http://github. $\mathrm{com} /$ jrnold/ggthemes.

Auguie, B. (2017). gridExtra: Miscellaneous Functions for "Grid" Graphics. R package version 2.3.

Grolemund, G., \& Wickham, H. (2011). Dates and Times Made Easy with lubridate. Journal of Statistical Software, 40(3), 1-25. Recuperado de http://www.jstatsoft.org/v40/i03/.

Henry, L. \& Wickham, H. (2020). purrr: Functional Programming Tools. Recuperado de http://purrr.tidyverse.org, https://github. com/tidyverse/purrr.

Lin, T., \& Robinson, D. (2020). gganimate: A Grammar of Animated Graphics. Recuperado de https://gganimate.com, https:// github.com/thomasp85/gganimate.

McKinnon, S. E. (2020). lemon: Freshing Up your 'ggplot2' Plots. $R$ package version 0.4.4. Recuperado de https://github.com/stefanedwards/lemon.

Milton, S. \& Wickham, H. (2014). magrittr: A Forward-Pipe Operator for R. R package version 1.5.

Nussbaumer, C. (2015). Storytelling with Data: A Data Visualization Guide for Business Professionals. John Wiley \& Sons: New York. 
Ooms, J. (2018). gifski: Highest Quality GIF Encoder. Recuperado de https://gif.ski/ (upstream), https://github.com/r-rust/gifski (devel).

Pebesma, E. (2018). Simple Features for R: Standardized Support for Spatial Vector Data. The R Journal, 10(1), 439-446, doi: https://doi.org/10.32614/RJ-2018-009

R Core Team. (2020). R: A language and environment for statistical computing. $R$ Foundation for Statistical Computing, Vienna, Austria. Recuperado de https://www.R-project.org/.

Rudis, B., Bolker, B. \& Schulz, J. (2017). ggalt: Extra Coordinate Systems, 'Geoms', Statistical Transformations, Scales and Fonts for 'ggplot2'. R package version 0.4.0. Recuperado de https://github. $\mathrm{com} / \mathrm{hrbrmstr} /$ ggalt

Slowikowski, K. (2020). ggrepel: Automatically Position Non-Overlapping Text Labels with 'ggplot2'. R package version 0.8.2. Recuperado de http://github.com/slowkow/ggrepel

Stengel, D., Calori, G. \& Giannoudis, P. (2008). Graphical data presentation. Injury, 39(6), 659-665.

Temple, D. (2020). XML: Tools for Parsing and Generating XML Within $R$ and S-Plus. $R$ package version 3.99-0.3. Recuperado de http://www.omegahat.net/RSXML

Wickham, H. \& Henry, L. (2020). tidyr: Tidy Messy Data. https:// tidyr.tidyverse.org, Recuperado de https://github.com/tidyverse/ tidyr.

Wickham, H. (2016). ggplot2: Elegant Graphics for Data Analysis. New York: Springer-Verlag.

Wickham, H. (2019). rvest: Easily Harvest (Scrape). Recuperado de http://rvest.tidyverse.org/, https://github.com/tidyverse/rvest.

Wickham, H. (2019). stringr: Simple, Consistent Wrappers for Common String Operations. Recuperado de http://stringr.tidyverse.org, https://github.com/tidyverse/stringr.

Wickham, H. (2020). forcats: Tools for Working with Categorical Variables (Factors). Recuperado de http://forcats.tidyverse.org, https://github.com/tidyverse/forcats.

Wickham, H., Hester, J., \& Ooms, J. (2020). xml2: Parse XML. Recuperado de https://xml2.r-lib.org/, https://github.com/r-lib/xml2. 
Wickham, H., François, R., Henry, L., \& Müller, K. (2020). dplyr: A Grammar of Data Manipulation. R package version 1.0.0. Recuperado de https://CRAN.R-project.org/package=dplyr

Zeileis, A., Fisher, J.C., Hornik, K., Ihaka, R., McWhite, C.D., Murrell, P., Stauffer, R., \& Wilke, C.O. (2019). colorspace: A Toolbox for Manipulating and Assessing Colors and Palettes. arXiv 1903.06490, arXiv.org E-Print Archive. Recuperado de http:// arxiv.org/abs/1903.06490. 


\section{Anexo 1. Lista de funciones disponibles en drcovidplots}

\begin{tabular}{|c|c|}
\hline Función & Descripción \\
\hline g_active_cases & Casos activos de COVID19 \\
\hline g_cases_province & $\begin{array}{l}\text { Provincias con la mayoría de los casos positivos de } \\
\text { COVID19 }\end{array}$ \\
\hline g_death & Número total de muertes \\
\hline g_density & $\begin{array}{l}\text { Correlación entre los casos de COVID19 y la densidad } \\
\text { poblacional }\end{array}$ \\
\hline g_evolution_covid & Evolución de casos nuevos y casos totales de COVID19 \\
\hline g_growth_cases & Tasa de crecimiento de casos positivos de COVID19 \\
\hline g_hospital & Personas en aislamiento hospitalario por COVID19 \\
\hline g_incidence & Incidencia de COVID19 \\
\hline g_incidence_region & Tasa de incidencia y letalidad por región de COVID19 \\
\hline g_12 & Tasa de letalidad (L2) de Ghani et al. (2005) de COVID19 \\
\hline g_log_nc & $\begin{array}{l}\text { Casos nuevos versus casos totales en logaritmos de } \\
\text { COVID19 }\end{array}$ \\
\hline g_map_covid & Mapa dominicano con casos de COVID-19 por provincia \\
\hline g_np & Casos negativos y positivos de COVID19 \\
\hline g_np_daily & Casos diarios negativos y positivos de COVID19 \\
\hline g_positive & Positividad nacional del COVID19 \\
\hline g_positive_province & Positividad por provincia de COVID19 \\
\hline g_positive_region & Positividad por región de COVID19 \\
\hline g_recovered & Recuperados de COVID19 \\
\hline g_recovered_daily & Número diario de personas recuperadas de COVID19 \\
\hline g_recovered_growth & Tasa de crecimiento de los recuperados de COVID19 \\
\hline g_recovered_province & Recuperado por provincia de COVID19 \\
\hline g_region & Casos COVID por región \\
\hline g_sex & Distribución por sexo de casos positivos de COVID19 \\
\hline g_status & $\begin{array}{l}\text { Distribución por estado de individuos afectados de } \\
\text { COVID19 }\end{array}$ \\
\hline g_tests & Pruebas de COVID19 \\
\hline g_world & Comparación mundial de la situación de COVID19 \\
\hline load_data_covid_dr & Carga las bases de datos \\
\hline
\end{tabular}


Anexo 2. Lista de paquetes utilizados para la creación de drcovidplots

\begin{tabular}{|l|l|}
\hline Paquete & Autor(es) \\
\hline ggplot2 & Wickham, H. (2016) \\
\hline dplyr & $\begin{array}{l}\text { Wickham, H., R. François, L. Henry \& K. Müller } \\
(2020)\end{array}$ \\
\hline tidyr & Wickham, H. \& L. Henry (2020) \\
\hline purrr & Henry, L. \& H. Wickham (2020) \\
\hline stringr & Wickham, H. (2019) \\
\hline magrittr & Milton, S. \& H. Wickham (2014) \\
\hline gganimate & Lin Pedersen, T. \& D. Robinson (2020) \\
\hline lubridate & Grolemund, G. \& H. Wickham (2011) \\
\hline XML & Temple Lang, D. (2020) \\
\hline rvest & Wickham, H. (2019) \\
\hline xml2 & Wickham, H., J. Hester \& J. Ooms (2020) \\
\hline grDevices & R Core Team (2020) \\
\hline ggalt & Rudis, B., B. Bolker \& J. Schulz (2017) \\
\hline ggthemes & Arnold, J.B. (2019) \\
\hline gridExtra & Auguie, B. (2017) \\
\hline grid & R Core Team (2020) \\
\hline forcats & Wickham, H. (2020) \\
\hline gifski & Ooms, J. (2018) \\
\hline colorspace & $\begin{array}{l}\text { Zeileis, A., J. C. Fisher, K. Hornik, R. Ihaka, C. } \\
\text { D. McWhite, P. Murrell, R. Stauffer, C. O. Wilke } \\
\text { (2019) }\end{array}$ \\
\hline ggrepel & Slowikowski, K. (2020) \\
\hline lemon & McKinnon, S.E. (2020) \\
\hline sf & Pebesma, E. (2018) \\
\hline
\end{tabular}

